This item was submitted to Loughborough's Research Repository by the author.

Items in Figshare are protected by copyright, with all rights reserved, unless otherwise indicated.

\title{
The sub-grid-scale approach for modelling the impingement cooling flow in the combustor pedestal tile
}

\section{PLEASE CITE THE PUBLISHED VERSION}

https://doi.org/10.1115/1.4038210

\section{PUBLISHER}

(C) American Society of Mechanical Engineers (ASME)

VERSION

AM (Accepted Manuscript)

\section{PUBLISHER STATEMENT}

This work is made available according to the conditions of the Creative Commons Attribution 4.0 Unported Licence (CC BY). Full details of this licence are available at: https://creativecommons.org/licenses/by/4.0/

\section{LICENCE}

CC BY 4.0

\section{REPOSITORY RECORD}

Ammour, Dalila, and Gary J. Page. 2017. "The Sub-grid-scale Approach for Modelling the Impingement Cooling Flow in the Combustor Pedestal Tile”. figshare. https://hdl.handle.net/2134/26349. 


\section{American Society of Mechanical Engineers}

SETTING THE STANDARD

\section{ASME Accepted Manuscript Repository}

\section{Institutional Repository Cover Sheet}

\begin{tabular}{lll}
\hline First & Last
\end{tabular}

The sub-grid-scale approach for modelling the impingement cooling flow in the combustor ASME Paper Title: pedestal tile

\section{Dalila Ammour and Gary J. Page}

Authors:

ASME Journal Title: Journal of Heat Transfer,

Volume/Issue _ 140(4): paper 042203

Date of Publication (VOR* Online)

$27^{\text {th }}$ Dec. 2017

ASME Digital Collection URL: $\underline{\text { http://heattransfer.asmedigitalcollection.asme.org/article.aspx?articleid=2658 }}$

DOI: $\quad$ https://doi.org/10.1115/1.4038210

*VOR (version of record) 


\title{
The Sub-Grid-Scale Approach for Modelling Impingement Cooling Flow in the Combustor Pedestal Tile
}

\author{
Dalila Ammour \\ Research Associate \\ Department of Aerospace and Automotive Engineering \\ Loughborough University \\ Loughborough LE11 3TU, UK \\ Email: d.ammour@lboro.ac.uk \\ Gary J. Page \\ Professor of Computational Aerodynamics Associate Dean for Research \\ Department of Aerospace and Automotive Engineering \\ Loughborough University \\ Loughborough LE11 3TU, UK \\ Email: g.j.page@lboro.ac.uk
}

The widely used gas turbine combustor double-walled cooling scheme relies on very small pedestals. In a combustor it is impractical for CFD to resolve each pedestal individually as that would require a very large amount of grid points and consequent excessive computation time. These pedestals can be omitted from the mesh and their effects captured on the fluid via a pedestal sub-grid scale (SGS) model. The aim is to apply the SGS approach, which takes into account the effects on pressure, velocity, turbulence and heat transfer, in an unstructured CFD code. The flow inside a 2-D plain duct is simulated to validate the pedestal SGS model and the results for pressure, velocity and heat transfer are in good agreement with the measured data. The conjugate heat transfer inside a 3-D duct is also studied to calibrate the heat source term of the SGS model due to the pedestals. The resolved flow in the combustor pedestal tile geometry is numerically investigated using RANS and LES in order to first assess the viability of the RANS and LES to predict the impinging flow and second to provide more validation data for the development of the SGS pedestal correlations. It is found that the complexity of such a flow, with high levels of curvature, impingement and heat transfer, poses a challenge to the standard RANS models. The LES provides more details of the impinging flow features. The pedestal model is then applied to the complete tile to replace the pedestals. The results are close to both the fully resolved CFD and the measurements. To improve the flow features in the impingement zone the first two rows were resolved with the mesh and combined with the SGS modelling for the rest of the tile, this gave optimum results of pressure, velocity and turbulence kinetic energy distribution inside the pedestal cooling tile.

\author{
Nomenclature \\ $A$ Area \\ $C_{p} \quad$ specific heat \\ $D$ diameter of pedestals \\ $f$ friction factor \\ $k$ turbulence kinetic energy \\ $L \quad$ length of the pedestal tile \\ $m$ mass flow rate \\ $\mathrm{Nu}$ Nusselt number \\ $P \quad$ local mean static pressure \\ $\mathrm{Pr}$ fluid Prandtl number \\ $q$ heat flux rate \\ $\mathrm{Re}$ Reynolds number \\ $\mathrm{Nu}$ Nusselt number \\ $S_{t} \quad$ traverse pedestal spacing \\ $S_{l} \quad$ axial pedestal spacing \\ $T$ mean temperature \\ $U_{i}$ mean velocity in i direction \\ $V$ velocity normal to pedestal axis \\ $W$ width of the pedestal tile \\ $x_{i} \quad$ Cartesian component \\ $\varepsilon$ turbulence dissipation rate \\ $\lambda$ thermal conductivity \\ $v$ fluid kinematic viscosity \\ $\rho$ density
}

\section{Introduction}

Targets in the design of gas turbine combustors are based on increasing the efficiency of the engine, minimising the production of the oxides of nitrogen $\left(N O_{x}\right)$ and maintaining 
an acceptable temperature at the exit of the combustor that can be tolerated by the turbine. Effusion cooling techniques are therefore important factors in improving efficiency of current engines. The prediction of the temperature and heat transfer throughout the solid material of a gas-turbine combustor has driven recent interest in cooling technology. An overview of various cooling styles used in combustion systems are described in Haselbach and Parker [4]. Effusion cooling is widely used and it offers high cooling effectiveness and full-coverage-film-cooling effect. An alternative to effusion cooling are pedestal tiles on double-skinned combustor liners; these are still of interest due to their simple design and low manufacturing cost, however, optimisation of different parameters such as cooling holes, pedestal arrangements and tile configurations are needed. Thorpe [15] investigated experimentally the combustor cooling tile geometry with the aim of exploring the flow distribution within the tile, on the Rolls-Royce Trent series of engines, to relate it to the cold-side cooling performance and establish appropriate design correlations. Thorpe provided a wide range of data which included velocity, pressure distribution and correlations for the friction factor and the heat transfer coefficient along the cold side of the tile. Completely resolving the substantial amount of cooling holes in combustor liners and the turbine blades using CFD is impractical. The prediction of combustor wall temperatures has traditionally been carried out using empirically based approaches such as that described by Lefebvre and Herbert [6]. A quasi-onedimensional model of the internal radiative and convective wall heat loading is coupled with empirical expressions for cold side cooling. It is generally accepted that these methods are valuable design tools, and are quick and cheap compared to CFD based methods, but their major drawback is that, due to their 1-D nature, they cannot predict the location or magnitude of any hotspots. Luff [7] suggested a Sub-Grid-Scale (SGS) approach to model the multiple smallscale cooling features which cannot be resolved by the computational mesh. Luff validated his method in a multi-block code against experimental data of Metzger [10]. He presented only results of pressure, velocity and turbulence. The assembled SGS correlations were used to predict the flow, thermal and stress fields in a geometrically complex combustor heatshield/backplate assembly by Luff and McGuirk [8]. The results showed that the SGS approach has significant value for the combustor design and life prediction. Denman et al. [11] investigated experimentally and numerically a simplified model of two overlaped cooling tiles, they applied the SGS approach of Luff [7] to the pedestal flow in a multi-block code, and showed only qualitative results of turbulence kinetic energy and temperature which suggested that the SGS approach performed well, they also mentioned that the pressure drop was overpredicted. The SGS correlations are also used to model the tube banks in heat exchangers. Zukauskas [16] suggested correlations with a comprehensive summary of experimental data on fluid flow and heat transfer for in-line and staggered long tubes. Sub-models similar to the one described above have also been used in the analysis of heat exchanges (see for example Pritbiviraj and An- drews [12]) where the flow is across banks of long tubes.

In this work, the pedestal SGS model approach is developed in an unstructured finite volume code using unstructured grid topologies. The SGS model is first validated for flow and conjugate heat transfer inside the 2-D and 3-D plain ducts of Metzger et al. [10]. Results of velocity, pressure and Nusselt number prediction are presented and compared with the available experimental data. The combustor pedestal cooling tile of Thorpe [15] is investigated using both RANS and LES in order to first assess the accuracy of RANS to predict the impinging flow and to provide additional validation data to calibrate the current pedestal SGS model. The combustor pedestal tile is then modelled using the SGS approach and the qualitative results of velocity, pressure and turbulence are presented and compared with CFD and experimental data. The latter results showed the model performs well. An additional work has been carried out to improve the performance of the SGS model by resolving two rows of pedestals in the impingement region.

\section{Turbulence modelling}

The Navier-Stokes and energy equations for incompressible flow are solved here. The following energy equation is solved in both the solid and the fluid regions to allow conjugate heat transfer:

$$
\frac{\partial U_{j} T}{\partial t}+\frac{\partial}{\partial x_{j}}\left(U_{j} T\right)=\frac{\partial}{\partial x_{j}}\left(\frac{\lambda}{\rho C_{p}} \frac{\partial T}{\partial x_{j}}-\overline{u_{j} t}\right)
$$

In the solid region, the velocity components are zero, so only the right-hand-side of Eqn. (1) is solved.

\subsection{RANS models}

The high-Re eddy-viscosity turbulence model used in this work is the $k-\varepsilon$ model of Launder and Spalding [5]. Two transport equations are solved, for the turbulent kinetic energy, $k$, and its dissipation rate, $\varepsilon$, the details such as coefficient values are given in Jones and Launder [5]. The realizable $k-\varepsilon$ model of Shih et al. [13] is also used here. The $k-\varepsilon$ models do not account for any near-wall viscous effects, and so are used with wall-functions (here, the 'two-velocity scale' standard wall-function). The modified $k-\varepsilon$ model by Durbin [2] is also used in some cases as a time scale limiter to remove the overprediction of $k$ in the stagnation flows. The low-Re eddy viscosity scheme tested is the shear stress transport model (SST) of Menter [9].

\subsection{Large Eddy Simulation}

The LES approach solves the unsteady N-S equations. The Smagorinsky sub-grid-scale model [14] is used to model the small scales in the present LES. The dynamic Smagorinsky model is also used and the results showed no significant changes when compared with the results obtained from the Smagorinsky SGS model with low Smagorinsky constant $\left(C_{S}\right.$ is taken as 0.1). The LES computation is initialised from a 
precursor converged unsteady RANS simulation. The mean profiles of the velocity from the RANS computation are those of a turbulent flow, and they can consequently allow the LES solution to develop more rapidly than if initialised from some other, more arbitrary field.

\section{The pedestal Sub-Grid-Scale method}

To fully resolve the flow in a complete pedestal tile geometry of a gas-turbine combustor liner would result in very large meshes and excessively long run times. Alternatively the pedestals can be omitted from the computational mesh and their effects on the fluid flow captured via a sub-gridscale (SGS) model of Luff and Mcguirk [7]. The development of the unstructured code towards implementation of the SGS pedestal model consists of several main steps:

1. Modelling of the additional pressure drop caused by hydraulic resistance of the pedestals/pins. This is achieved by adding static pressure gradients in the momentum equations:

$$
R_{x i}=0.5 \frac{1}{S_{l}} \rho f_{p e d} V_{x i, \max } V_{\max }
$$

Where $R_{x i}$ is the extra pressure gradient term, $S_{l}$ and $S_{t}$ are the axial and traverse pitches respectively, $V$ is the velocity component normal to the pedestal's axes, $D$ is the diameter of the pins, $V_{\max }=V\left(S_{t} /\left(S_{t}-D\right)\right)$ and $f_{\text {ped }}$ is the friction factor defined from the tube bundle correlation of Zukauskas [16].

2. Pedestal arrays in the combustor liner enhance the production and dissipation of turbulence kinetic energy. By comparison with turbulence generation at a wall, the turbulence production is given by the product of the wall shear stress and the velocity parallel to the wall. Assuming that the total pressure loss due to the work done overcoming the blockage effect of pedestal array is a source of turbulence kinetic energy which is dissipated into internal energy by (See Pritbiviraj and Andrews [12]). Modelling of the additional turbulence kinetic energy (TKE) and dissipation rate is achieved by adding more terms:

Turbulence:

$$
R_{k}=\frac{1}{\rho} \sum_{i} R_{x i} V_{x i}
$$

Dissipation:

$$
R_{\varepsilon}=C_{\varepsilon 1} \frac{\varepsilon}{k} R_{k}
$$

Where $R_{k}$ is the extra turbulent kinetic energy term. $R_{\varepsilon}$ is the extra dissipation rate term.

3. Modification of the existing energy Eqn. (1) to model the conjugate heat transfer in the solid and the fluid regions. The same energy equation is solved for the solid region, with the exception that the velocity components are set to zero. An additional source term $S_{T}$ is added to the discretised energy Eqn. (1) for cells in the pedestal region:

$$
S_{T}=\frac{N u \lambda}{D} A_{p e d}\left(T_{p e d, P}-T_{P}\right)
$$

Where $\lambda$ is the effective (molecular+turbulent) heat diffusivity, it is equal to thermal solid material conductivity in the solid region and is equal to thermal diffusivity in the fluid region. $A_{p e d}$ is the effective pedestal surface area in the cell, defined as: $A_{p e d}=\pi * D / S_{t} * S_{l}$. $T_{p e d, P}$ is the temperature of the fluid in the pedestal region defined as: $T_{p e d, P}=T_{p e d}-T_{P} ; T_{p e d}$ is the pedestal (solid) temperature and $T_{P}$ is the temperature of the fluid in the cell. The Nusselt number $(\mathrm{Nu})$ in Eqn. (5) is calculated from experimentally derived correlations of Zukauskas [16] for heat transfer staggered tube bundles in the cross-flow:

$$
N u_{\text {ped }}=0.35{\frac{S_{t}}{S_{l}}}^{0.2} \operatorname{Re}_{\text {ped }}^{0.6} \operatorname{Pr}^{0.36}
$$

Where $\operatorname{Pr}$ represents the Prandtl number evaluated at the bulk mean temperature, it is taken as 0.7 based on Luff and McGuirk [8].

It is important to note that careful attention has to be paid to the heat flux evaluation at the fluid/solid interface. The fact that the diffusion coefficient changes sharply across a fluid/solid interface means that discontinuous temperature gradients may results. More advanced approaches such as the generalise gradient diffusion hypothesis (GGDH) can also be used to model heat fluxes in Eqn. (1). A numerical scheme has also been developed by Luff [8] to tackle problems associated with non-orthogonality of the mesh for more complex geometries.

\section{Numerical methods}

The flow solver is the Rolls-Royce unstructured code Precise-uns [1], which is based on a 3-D, cell-centred, finite volume, implicit solution of the Navier-Stokes equations in either Reynolds-averaged or LES forms. The SIMPLE approach is used to couple the pressure and the velocity fields for incompressible flows, more details of this method is explained in Ferziger [3]. Grid sensitivity tests have been carried out to verify the accuracy of the results. Unstructured non-uniform grids, generated with ANSYS ICEM-CFD, are used. The boundary layer is resolved using prisms. The computational mesh used for the LES is unstructured and non-uniform, it contains around 8 million elements, with clustering in the near wall region to obtain non-dimensional near-wall node distances of around 1. The core grid spacing in wall units also satisfies the constraints $\triangle x^{+}<40$ and $\triangle z^{+}<20$ 
Among the six convection schemes embedded in the code, the central differencing scheme is used for the LES simulation and the second order three level scheme, is used for the time discetisation and the time step is $\delta t=10^{-3} s$, resulting in a CFL less than 1 for the majority of the flow. The LES simulation of the flow in the pedestal tile required 30,000 time steps, 30s real time and approximately 500 hours CPU time in 16 CPU's, to reach a fully turbulent flow state, and at least a further 30,000 time steps for statistical averaging of the flow field. For the steady RANS simulation, the secondorder linear upwind scheme is used for reason of stability and the first-order Euler scheme is used for the time discretisation. A coarse non-uniform mesh of 500,000 elements is generated for high-Re models. The SST model needs a well resolved mesh near the wall.

\section{Flow in 2-D plain duct}

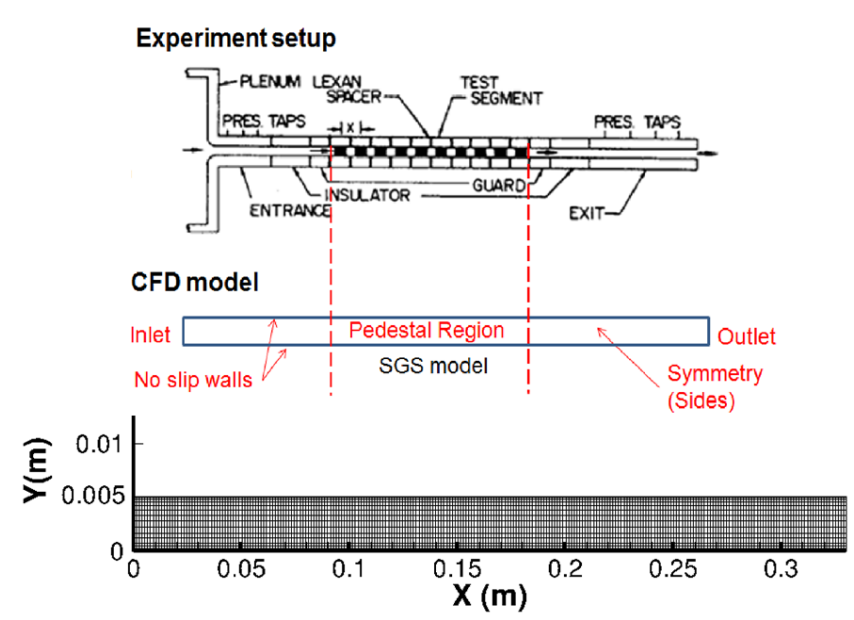

Fig. 1. Experiment setup [10], CFD geometry, mesh and boundary conditions of the 2-D duct.

In order to validate the pedestal SGS model approach, tests were made on the plain duct of Metzger et al. [10] for a Reynolds number $(\mathrm{Re})$ of $10^{5}$, where $\mathrm{Re}$ is based on the diameter of the pedestals $D$ and $\bar{V}_{\text {max }}$, the bulk average velocity in the minimum flow area between pedestals. First, only the momentum and the turbulence are initially considered. The geometry, mesh and boundary conditions of the plain rectangular duct are presented in Fig. 1. A narrow slice of the duct in the traverse direction is modelled, the geometry is reduced to 2-D and symmetry boundary conditions were applied at the spanwise sides of the domain. Results are compared with the experiment of Metzger et al. [10]. The geometry consists of a long entrance section to allow the flow to be fully developed, the pedestal region where the SGS model approach is embedded and an exit section added at the right end of the duct. The grid used for both the plain duct and the pedestal SGS model computations is structured hexahedral, non-uniform and clustered near the wall.

The normalised static pressure $\left(\left(p-p_{\text {inlet }} / p_{\text {inlet }}\right) \times 100\right)$ pro- file along the streamwise line of the duct at mid-height is shown in Fig 2. When comparing the pressure distribution along the plain duct with a duct with the embedded pedestal SGS approach, it can be seen that the pressure gradient in the pedestal region is very much greater than that in the entrance and exit, while the pressure gradient trend for the plain duct without the SGS model is rather more flat indicating a smaller pressure gradient. The current results are in good agreement with the data of Metzger et al. [10] and also reported in Luff [7].

In Figure 3, the predicted fully developed normalised velocity $\left(V / V_{0}\right)$ profile along the height of the plain duct (no pedestals) and the duct with the pedestal SGS model are plotted at position $\mathrm{X} / \mathrm{L}=0.7$. It can be seen that the pedestal SGS model has the effect of flattening the velocity profile considerably, and this means that the effective boundary layer thickness is reduced. The results are very close to the experimental data presented in Luff [7].

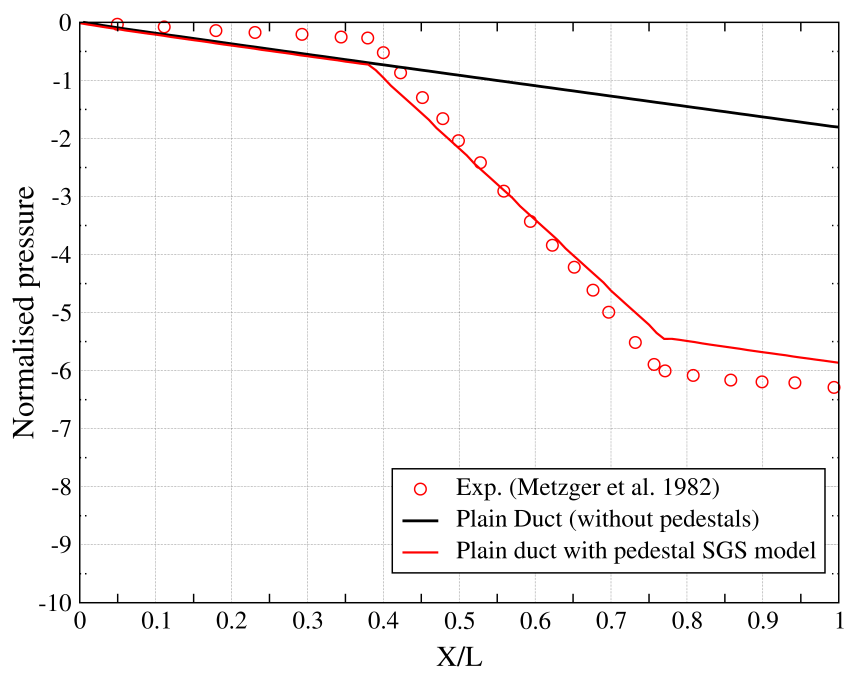

Fig. 2. Normalised static pressure profiles at the mid-height of the duct.

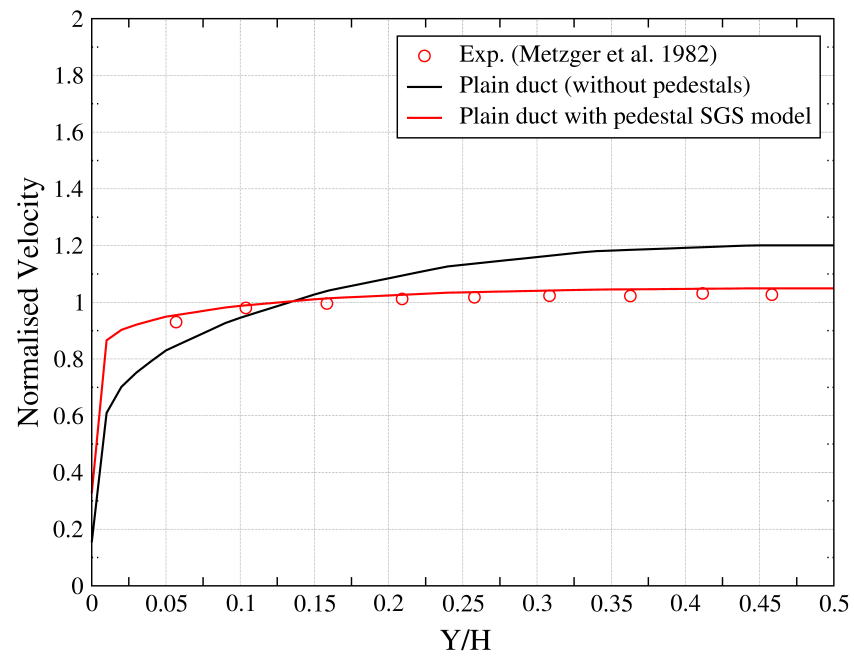

Fig. 3. Normalised streamwise velocity profile along the height of the duct at $\mathrm{X} / \mathrm{L}=0.7$. 


\subsection{Flow and conjugate heat transfer in 3-D duct}

Conjugate heat transfer tests were carried out on the plain duct of Metzger et al. [10] in order to validate the pedestal SGS heat source term. First, the flow in the 3-D duct is computed to provide additional data to the one presented in Luff [7]. The geometry is a rectangular duct containing staggered arrays of ten rows of pedestals. The pedestal region is preceded by a long entrance section. The pedestals and the upper and lower duct walls in the pedestal region are made of copper. The side walls and the walls in the entrance and exit sections are made from insulating material (wood). The upper and lower walls of the pedestal region are heated and maintained at a temperature of $320 \mathrm{~K}$, and the temperature of the fluid flow is $300 \mathrm{~K}$. The 3-D duct is investigated numerically for a Reynolds number of $10^{4}$, using a well resolved mesh to model the conjugate heat transfer and to define correct parameters, such as the temperature in the pedestal region and at the exit of the duct, these parameters are added in Eqn. (5) and are taken into account for the development of the pedestal SGS approach. Prior to this computation, a grid sensitivity test was carried out in order to test the difference between the hexahedral and mixed elements tetrahedral/prism grids to compute the impinging flow across rows of tubes. Results of pressure velocity and temperature showed no difference between both meshes but it is important to note that for the hexahedral grid the element skewness can be high if the mesh is refined further especially around the tubes. The mesh chosen to be used is then mixed elements and contains around 4 million elements with clustered hexa prism layers near the wall giving $y^{+}$less than 1 . The ge-
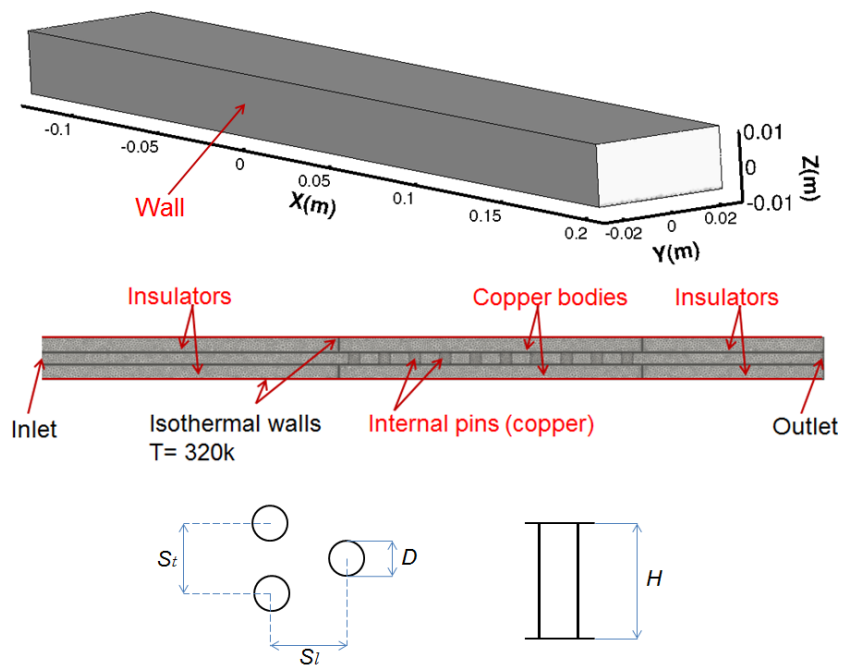

Fig. 4. Geometry, mesh and boundary conditions of the 3-D duct.

Table 1. Geometric parameters of the 3-D duct.

\begin{tabular}{lllll}
\hline & $\mathrm{D}(\mathrm{mm})$ & $S_{t} / D$ & $S_{l} / D$ & $\mathrm{H} / \mathrm{D}$ \\
\hline 3-D duct & 5.08 & 2.5 & 2.5 & 1 \\
\hline
\end{tabular}

ometry, the computational mesh and the boundary conditions are shown in Fig. 4. The geometric parameters are illustrated in Table 1.

Results of temperature inside the 3-D duct show that the effects of the side walls are minimal on the temperature distribution, the temperature does not change significantly when moving from the side walls to the core of the duct. The heat is conducted from the upper wall through the copper walls to the pedestals giving a uniform temperature of $320 \mathrm{~K}$ (Fig. 5). The thermal field also shows that the flow entering from the left side of the duct picks up a certain bulk temperature when it interacts with the heated pins at $320 \mathrm{~K}$, in the pedestal region, giving an average temperature of $310 \mathrm{~K}$ at the exit section of the 3-D duct. Modelling the conjugate heat transfer inside the 3-D duct allowed us to define the approximate temperature in the pedestal region and at the exit section. The latter are embedded in Eqn. (5) for the validation of the SGS heat source term. Furthermore, for the purpose of validation of the SGS model and in particular the heat transfer source term, the computational analysis of the flow inside a

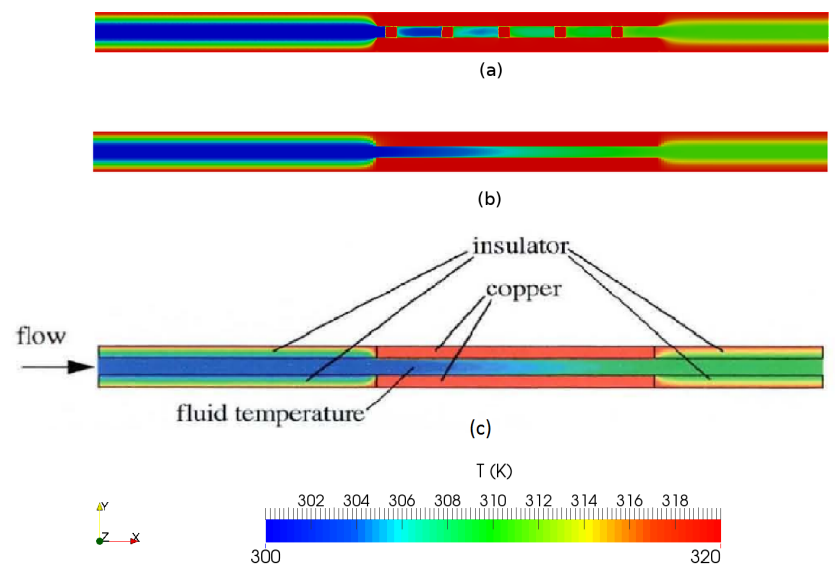

Fig. 5. Temperature contours at the vertical mid-section. (a) Duct with resolved pedestals. (b) SGS modelled plain duct. (c) SGS modelled plain duct (LUFF [7] MULTI-BLOCK CODE).

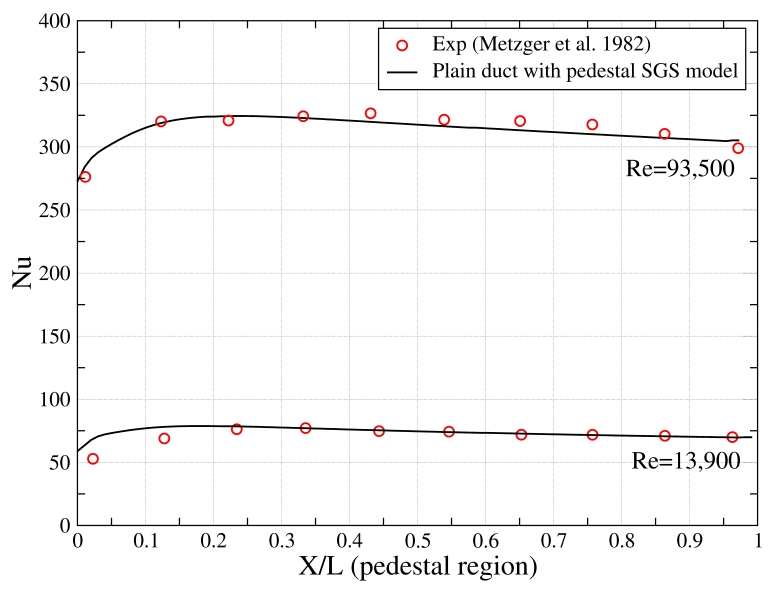

Fig. 6. Nu development along the streamwiseline of the 2-D duct. 
2-D duct is conducted. It was mentioned earlier that the side walls effects were minimal. The same geometry described in the previous paragraph is simulated but with symmetry boundary conditions at the side walls. The calculations were run at the same conditions and Reynolds number. Now, the pedestals are omitted from the mesh and are replaced by the pedestal SGS approach described earlier to account for the pedestals effects on pressure drop, turbulence generation and heat transfer. The results of temperature distribution at the vertical mid-plane of the 2-D duct are shown in Fig. 5-b. The temperature distribution inside the 2-D duct with the pedestal SGS model gives very close agreement with the 3-D fully resolved duct containing pedestals in Fig. 5-a. The temperature in the pedestal region and at the exit of the duct are in good agreement with the the temperature distribution in the 3-D duct showing that the pedestal SGS approach works well and correctly produces the effects of the pedestal temperature in the entire duct. The temperature predictions are also consistent with the results of Luff [7] in Fig. 5-c.

For further validation of the SGS heat source term, the Nusselt number results are compared with the experimental data of Metzger et al. [10]. The heat transfer coefficient (HTC) is defined here in terms of the heated surface heat flux and the difference between the heated surface temperature $(320 \mathrm{~K})$ and the reference temperature which is defined as the local bulk average temperature of the zero flux surface temperature. Figure 6 shows the variation of the Nusselt number across the streamwise line of the 2-D duct for two Reynolds numbers, $1.39 \times 10^{4}$ and $9.35 \times 10^{4}$. The results of the SGS model show that the $\mathrm{Nu}$ is slightly higher in the first half of the pedestal region which corresponds to the the first three to five rows in the experiment, it then decreases gradually through the remainder of the pedestal region. The $\mathrm{Nu}$ is highly dependent with the Reynolds numbers. The Nu results are in good agreement with experimental data of Metzger et al. [10] and are also consistent with Zukauskas [16] who reported increases in inner row heat transfer in tube banks.

\section{LES and RANS of the impinging pedestal tile flow for the validation of the pedestal SGS model}

The impinging flow across a pedestal tile is investigated numerically to assess the development of the pedestal SGS approach. Three steady RANS models, the standard $k-\varepsilon$, the realizable $k-\varepsilon$ and the SST $k-\omega$ have been used. The unsteady LES approach is also used to assess the accuracy of RANS simulations and to provide additional data for the development of the pedestal SGS approach. The pedestal tile test case and computational mesh are shown in Fig. 7 and Fig. 8, respectively. The characteristics of the mesh and the CPU time used for RANS and LES simulations are summarised in Table 2. The flow (air) enters the computational domain through a plenum via a feed-hole with a diameter of $0.027 \mathrm{~m}$. The mass flow and the temperature at the inlet are $0.0112 \mathrm{~kg} / \mathrm{s}$ and $333 \mathrm{~K}$ respectively, as in the experiment of Thorpe [15]. The tile is represented as an isothermal wall. The experiment showed that the flow is equally split, the geometry is then cut through the half of the feed-hole and a

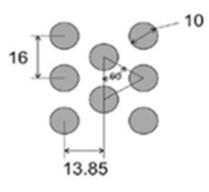

10

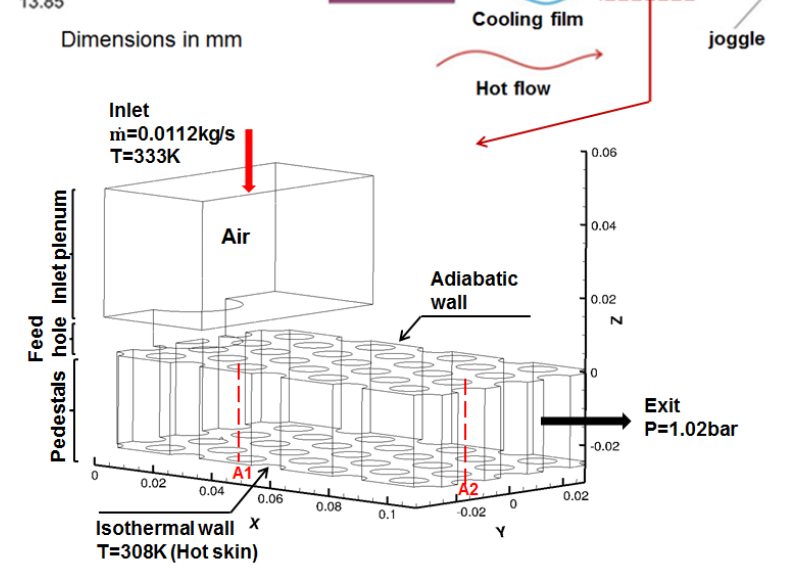

Fig. 7. Geometry, Dimensions and boundary conditions of the pedestal tile.

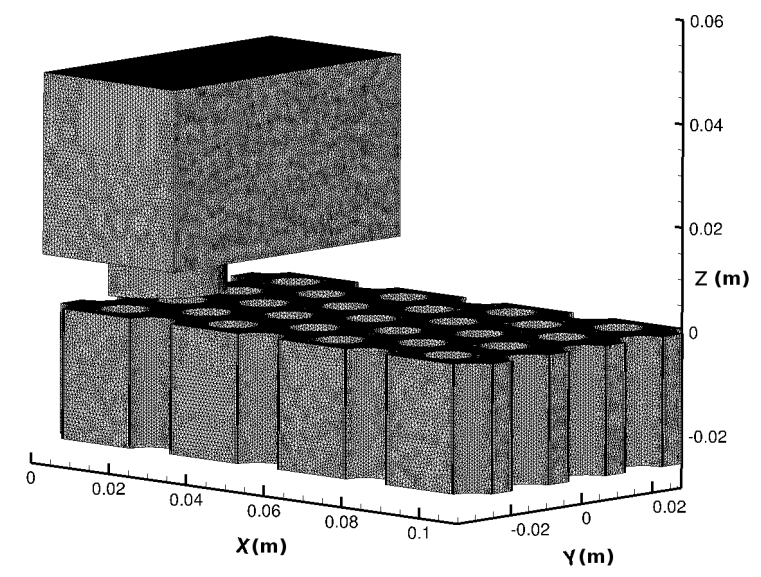

Fig. 8. Computational mesh of the combustor pedestal tile.

symmetry boundary condition is applied at the cutting plane. It is important to note that for LES the instantaneous flow is not symmetric but to reduce the model size and the computational cost, the symmetry boundary condition is still used. Periodicity boundary conditions are applied at the spanwise sides. The experiment showed that the flow physics do not change significantly and the fluid flow becomes evenly distributed after the sixth row when considering the full tile with 13 rows, and therefore only seven rows are considered in the CFD computation. Two vertical velocity and hot skin pressure plotting probes are shown in Fig. 7.

The temporal evolutions, at the probe location (), of the four global parameters, normalised pressure and normalised velocities obtained from unsteady LES and RANS are presented in Fig. 9 and Fig. 10 respectively. The profiles show periodic oscillations of pressure and velocities at the probe located in the impingement region where that are recirculations and many flow features.

It is found that the predicted pressure drop coefficient $\left(\triangle P_{\text {tile }} / P\right)$ needed to drive the flow field from the feeding 
Table 2. Characteristics of the mesh and CPU time of LES and RANS computations of the combustor pedestal tile.

\begin{tabular}{cllll}
\hline Case & LES1 & LES2 & SST & Realizable $k-\varepsilon$ \\
\hline Number of elements & 15 million & 8 million & 4 million & 1,5 million \\
Normalised CPU (16 cores) & 1 & 0.6 & 0.3 & 0.1 \\
$y^{+}$(near the wall) & $<1$ & $<1$ & $<1$ & $>30$ \\
$\triangle y^{+}$(core) & $<30$ & $<50$ & $<80$ & $<100$ \\
$\triangle y^{+}$(impingement region) & $<20$ & $<20$ & $<30$ & $<50$ \\
\hline
\end{tabular}

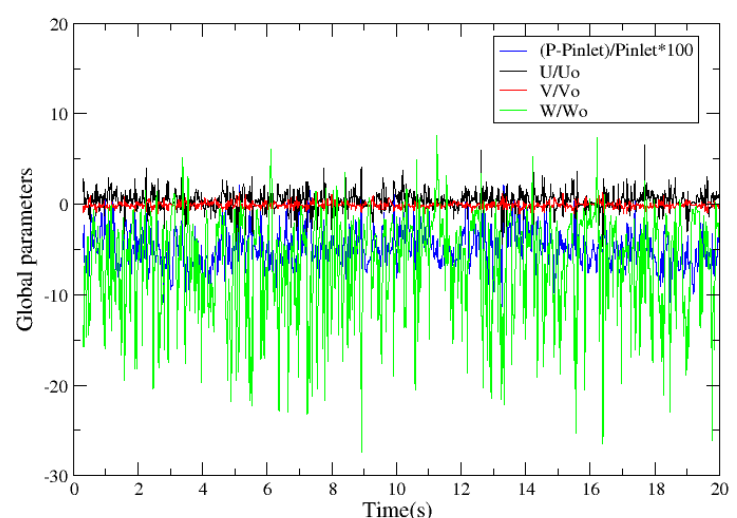

Fig. 9. Temporal evolution of LES.

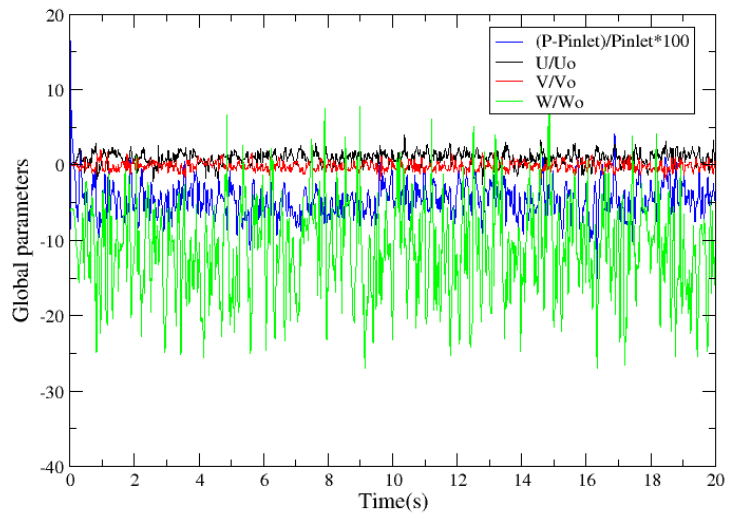

Fig. 10. Temporal evolution of unsteady RANS (SST).

plenum to the exit of the pedestal tile is around $2.47 \%$ which is very close to the experimental value $(2.5 \%)$.

Contours of instantaneous and time averaged normalised velocity magnitude $\left(V / V_{o}\right)$, where $V_{o}=m / \rho A$ is the reference velocity, along the horizontal cross-section at $\mathrm{Z} / \mathrm{H}=-0.5$ are shown in Fig. 11. The instantaneous velocity field obtained from LES (8 million elements), in Fig. 11-a, shows many small scale unsteady features which are more noticeable in the impingement region because of the high pressure gradient and the stagnation with the pedestals. The flow tends to have a repeating pattern and it is evenly distributed after the fourth row where it is independent of the feed-hole. Contours of time-averaged velocity magnitude, in Fig. 11-b confirms that the velocity is high at the impingement region due to

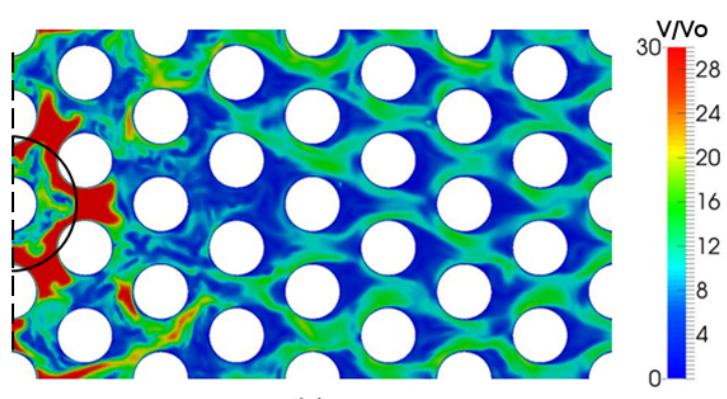

(a)

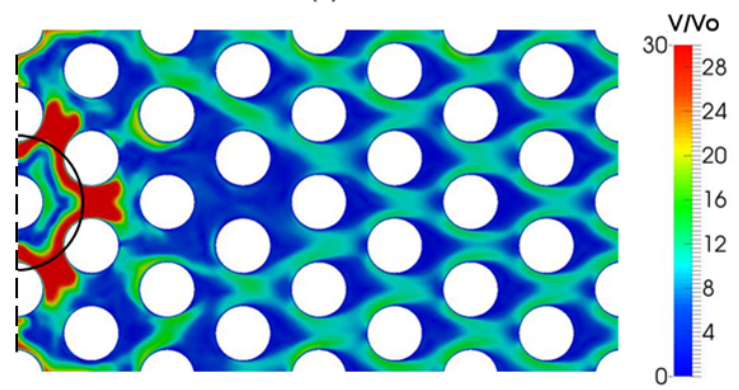

(b)

Fig. 11. Normalised velocity magnitude contours at the horizontal mid-section, LES results. (a) instantaneous, (b) time-averaged.

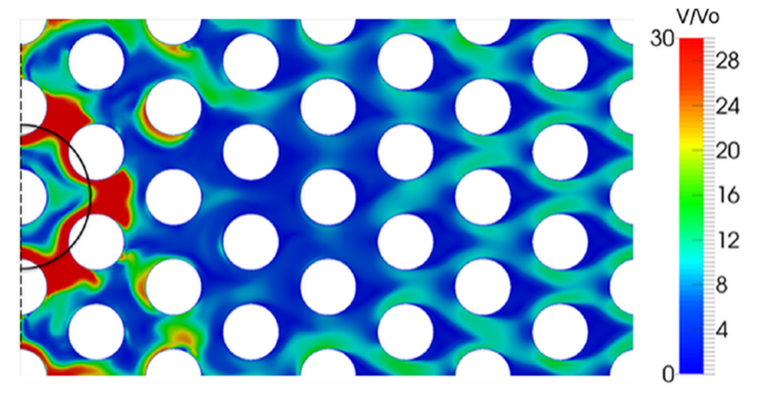

Fig. 12. Normalised velocity magnitude contours at the horizontal mid-section, SST results. 
the blockage effects of the impinging flow compared to the rest of the tile. The velocity contours obtained from the SST simulation, presented in Fig. 12, show similar high velocity magnitude in the impingement region and lower velocity in the rest of the tile however the SST model does not capture as much detail as the LES near the feed hole (the impingement region).

The normalised velocity magnitude comparisons between the RANS, two LES ( 8 milliom and 15 million) and the available data of Thorpe [15], at two probes A1 and A2 (see Fig. 7), are shown in Fig.13. At the impingement area (the second row, probe A1) the velocity magnitude is higher where there is strong adverse pressure gradients. Two LES calculations were carried out to verify the mesh sensitivity on the acuracy of the calculation. No significant differences have been noticed between the two LES calculations. This implies that 8 million cell elements are enough to resolve

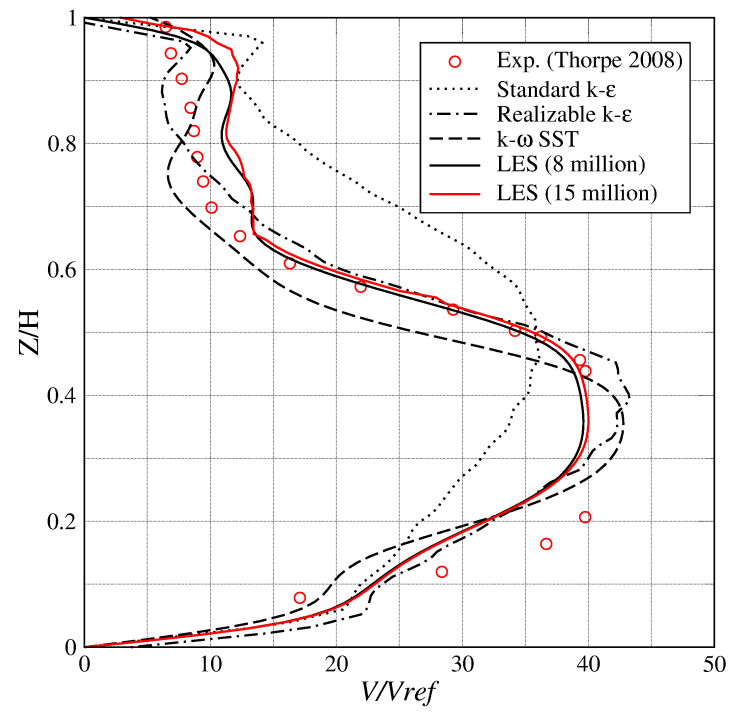

(a)

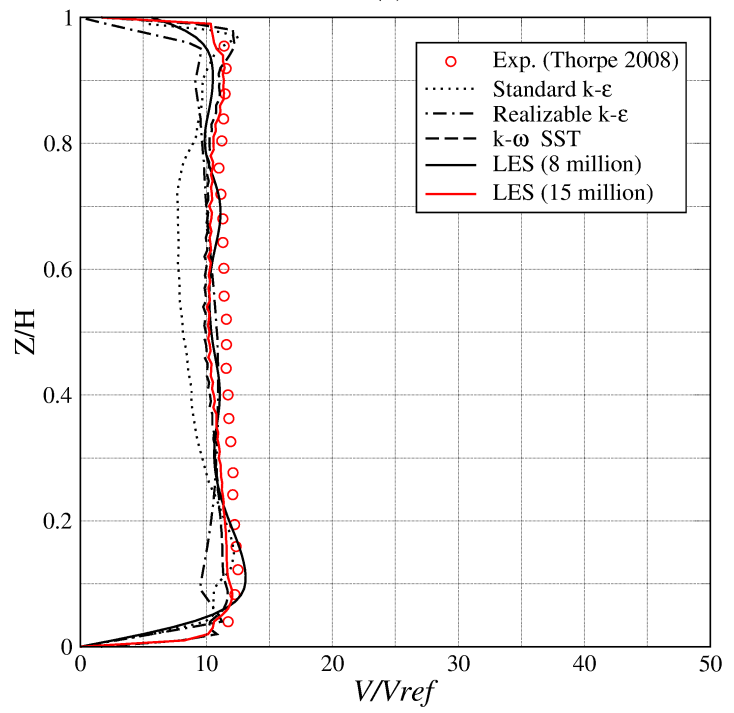

(b)

Fig. 13. Normalised velocity profiles at two probes inside the tile. (a) probe A1, (b) probe A2. the large scales flow features in the flow. The standard $k-\varepsilon$ model fails to capture the correct radial position of the jet and it underestimates the jet velocity. This is due to its limitation to predict impinging flows and due to the use of the standard wall-treatment to model the near wall shear stress. The realizable $k-\varepsilon$ is closer to the measured data due to the use of an enhanced wall treatment for impinging flows. The $k-\omega$ SST model and the LES results, with much more grid elements and well-resolved near wall spacing (mesh guidelines in Table 2), show better performance to capture the radial jet due to their ability to correctly resolve the shear stress near the wall. Profiles of velocity at probe A2 (near the exit of the tile) show similar velocity trends for all RANS models but the LES and the SST model shows better agreement with the measured data. The normalised velocity magnitude is constant along the height of the tile, this confirms previous findings which states that the flow is fully developed and more settled far from the feed-hole.

The heat transfer coefficient (HTC) is calculated as:

$$
h=\frac{q_{w}}{\Delta T}
$$

Where $\Delta T$ is the difference in temperature between the solid wall and the surrounding fluid area, in K. $q_{w}$ is the wall heat flux per unit area, in $\left(W / m^{2} k\right)$, obtained from the Fourier's law:

$$
q_{w}=-\lambda \frac{\partial T}{\partial x_{n}}
$$

Where $x_{n}$ denotes the direction normal to the wall.

The HTC results obtained from RANS and LES are compared with the experimental data of Thorpe [15] at the location $\mathrm{Y}=0.027 \mathrm{~m}$ along the hot skin of the pedestal tile. Contour plots and quantitative results of the HTCs are shown in Fig. 14 and Fig. 15 respectively. Figure. 14 shows qualitative streamwise representation of the HTC contours along the hot skin and comparison between a range of RANS models and the LES. High levels of HTCs are observed near the feed-hole, which are regions of high flow velocity, strong flow turning and stagnation, as stated above. The HTCs appears to settle to a uniform condition after typically four rows of pedestals, with a repeating pattern in subsequent pedestal rows. The RANS models predict correctly the general trend of the HTC distribution on the hot plate of the pedestal tile. The SST model is more in line with the LES and it captures the heat transfer distribution as it was observed in the experimental rig [15] in Fig. 14-a. The quantitative results of the HTC, in Fig. 15, show peaks of HTC along the hot plate caused by stagnation with the pedestals, these peaks are noticed to be higher in the impingement region where the velocity and pressure gradient are also high. The LES and the SST model capture most of the HTC peaks at the stagnation points while the $k-\varepsilon$ models underestimate slightly the HTC peaks at the half end of the hot plate because of the wall-function limitation to predict the correct heat flux when the wall shear stress falls to zero at stagnation regions. 


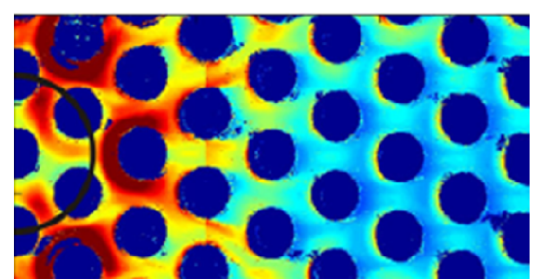

(a)

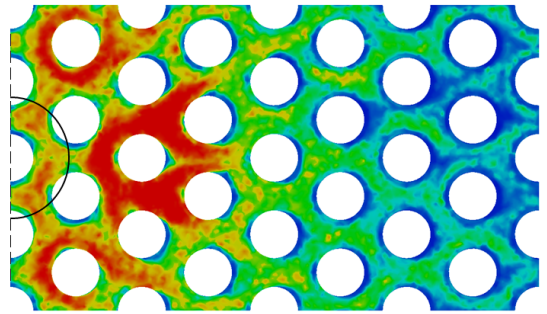

(c)

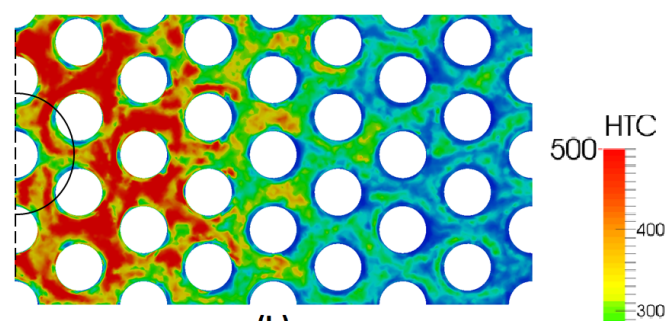

(b)

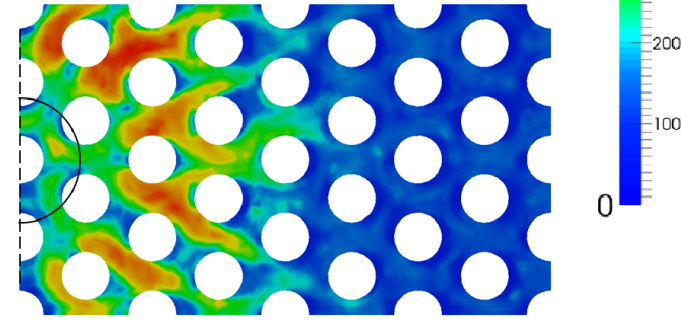

(d)

Fig. 14. Contour plots of HTCs at the hot surface of the tile. (a) Exp. of Thorpe [15], (b) LES, (c) SST, (d) Realizable $k-\varepsilon$.

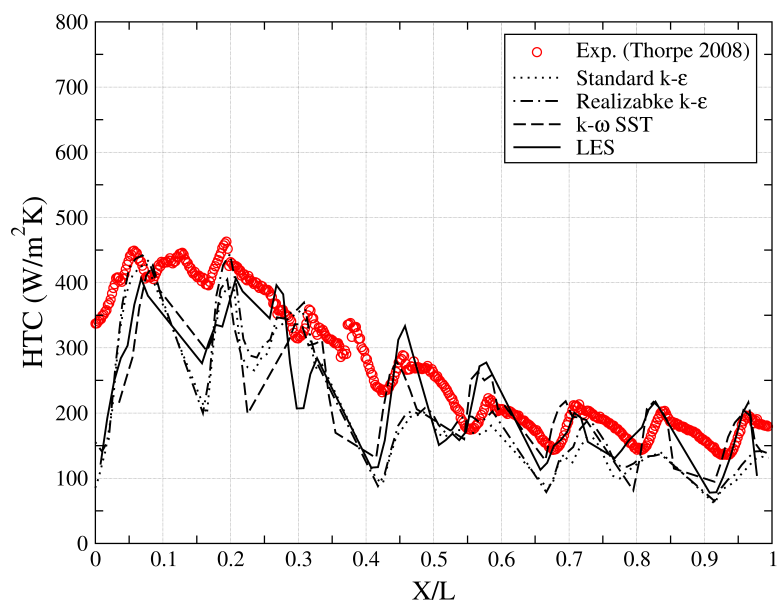

Fig. 15. Profiles of streamwise averaged HTCs along the hot plate at $\mathrm{Y} / \mathrm{W}=0.85$.

\section{SGS modelling of the combustor pedestal tile}

For further validation of the pedestal SGS approach, tests are carried out on the combustor pedestal tile, two CFD models are considered: Case 1, where all the pedestals are omitted from the tile and are replaced by the pedestal SGS model (momentum and turbulence source terms), and Case 2 , where only two rows of pedestals are resolved with the mesh and the rest of the pedestals are replaced by the SGS model. The aim is to model a more complex geometry such as the combustor pedestal tile using the SGS approach described above, and to compare the results with the available experimental data [15] and with the above CFD results.

The SGS pedestal model is used in conjunction with the realizable $k-\varepsilon$ model which uses coarse and wall-function. The pedestal SGS approach reduced the cost of the simulation by almost the half. The computing time is also reduced when resolving the first two rows near the feed-hole. Details of the mesh and computing time are presented in Table 3.

The quantitative results of the normalised static pressure are presented in Fig. 16. The pressure is plotted along the middle streamwise line of the hot impingement plate. The results of the normalised static pressure obtained from the realizable $k-\varepsilon$ model with the SGS approach for both Case 1 and Case 2 are compared with the the experimental data of Thorpe [15], LES and RANS. The results show high pressure in the impingement region then a gradual decrease of pressure towards the exit of the tile agreeing with the experiment and LES, however some discrepancies are noticed up to $\mathrm{X} / \mathrm{L}=0.2$ (first two rows), this indicates that the pedestal SGS model cannot capture the flow patern near the feed-hole. On the other hand, the overall pressure distribution in Case 2 show good agreement with the experimental data and LES

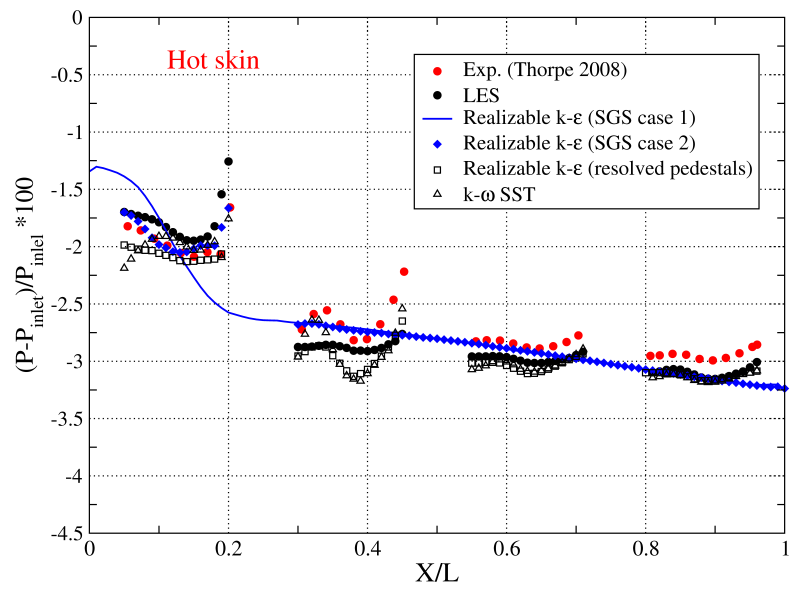

Fig. 16. Normalised static pressure profiles along the hot surface. 
Table 3. Characteristics of the mesh and CPU time of the SGS model.

\begin{tabular}{clll}
\hline Case & Realizable $k-\varepsilon$ & Realizable $k-\varepsilon$ (SGS Case1) & Realizable $k-\varepsilon$ (SGS Case2) \\
\hline Number of elements & 1,5 million & 500,000 & 700,000 \\
Normalised CPU (16 cores) & 1 & 0.4 & 0.6 \\
$\triangle y^{+}$(impingement region) & $<50$ & $<50$ & $<30$ \\
\hline
\end{tabular}
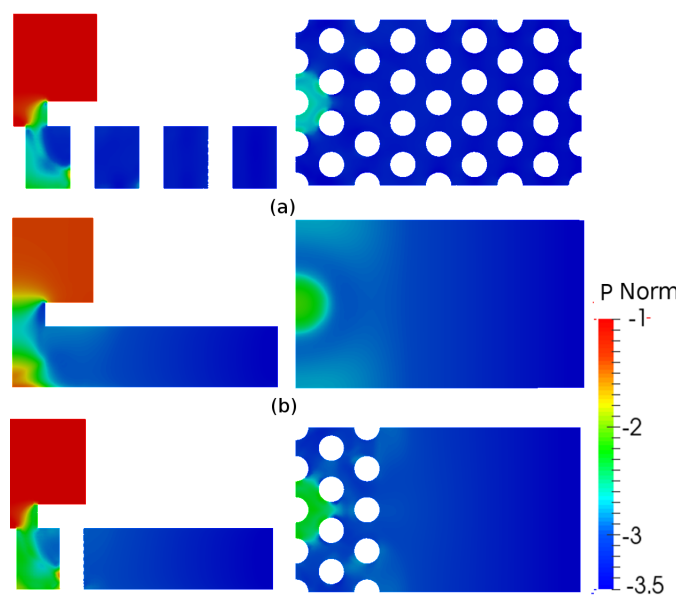

(c)

Fig. 17. Normalised pressure contours. pedestals, (b) Case 1, (C) Case 2.

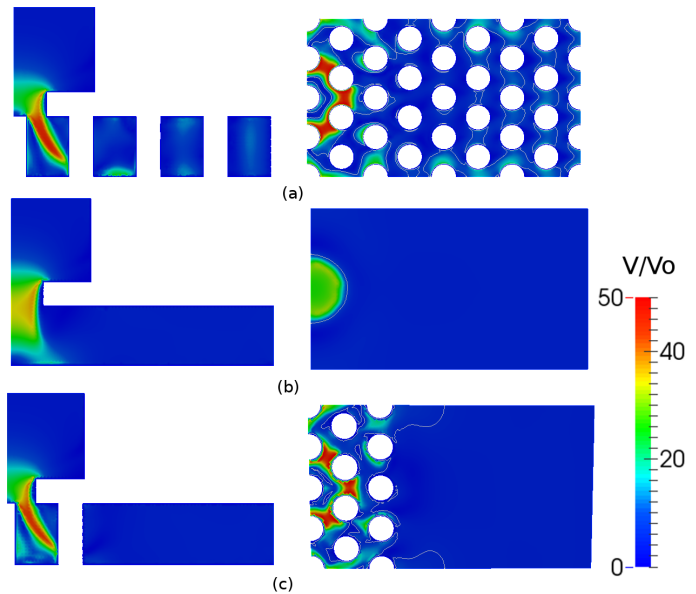

Fig. 18. Normalised velocity magnitude contours. (a) tile with resolved pedestals, (b) Case 1, (c) Case 2.

in the impinging region. This suggests that it is preferable to resolve at least two rows of pedestals individually using the computational mesh near the feed-hole, while the rest of the tile, where the flow is more settled, it can be modelled using the pedestal SGS model.

The qualitative results of normalised pressure, in Fig. 17, show close agreement between the resolved pedestals using the computational mesh (Fig. 17-a) and the modelled

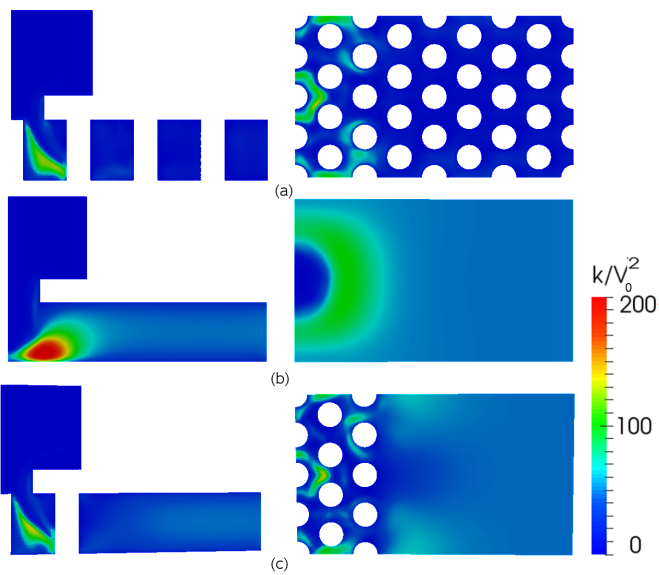

Fig. 19. Normalised TKE contours. (a) tile with resolved pedestals, (b) Case 1, (c) Case 2.

pedestals using the SGS model (Fig. 17-b). Some discrepancies were noticed in the impingement region when comparing the resolved pedestals and Case 1, Results of pressure of Case 2 (Fig. 17-c) show better agreement with the resolved tile than Case 1, this confirms the comments above.

The qualitative results of the normalised velocity magnitude inside the pedestal tile is shown in Fig. 18. The velocity distribution is generally higher near the feed-hole then it decreases gradually in the rest of the tile as mentioned earlier. The SGS model predicts well the overall flow pattern. The velocity near the feed-hole in Case 2, in Fig. 18-c, is better predicted than Case 1, in Fig. 18-b, when compared with the resolved pedestals in Fig. 18-a. This can be attributed to the fact that in Case 2 the first rows of pedestals are resolved so the blockage effect is captured in the impingement region which gives a higher velocity magnitude. This suggests that the velocity effects are well captured by the SGS model and the results are improved when the first two rows are resolved individually with the mesh.

The SGS model also takes into account the work provided by the pedestals in the form of turbulence kinetic energy (TKE). The qualitative results of the normalised TKE inside the pedestal tile, in Fig. 19, show higher turbulence near the feed-hole where the flow impinges generating more turbulence, The TKE is lower in the rest of the tile. In Case 1 (Fig. 19-b) the turbulence is slightly overpredicted inside the tile while in Case 2 (Fig. 19-c) the TKE is in close agreement with the resolved pedestal tile (Fig. 19-a). Again the SGS model performs better away from the feed-hole. 


\section{Conclusions}

The pedestal SGS model has been enhanced and embedded in an unstructured code to account for the effects of the pedestals in the combustor cooling tile. The latter model is validated for 2-D and 3-D plain ducts against available measurements. The model performed well, capturing the effects of momentum, turbulence and heat source due to the pedestals. The conjugate heat transfer is also computed to enhance the SGS model and for the prediction of the heat transfer in both the solid and the fluid regions of the pedestal tile. The combustor cooling tile was investigated numerically using both RANS and LES in order to assess the development of the pedestal SGS approach. Results of pressure, velocity and heat transfer were compared to experimental data and presented in this paper. LES showed many flow unsteady features near the feed-hole. These features are caused by the impingement and high pressure gradients. The flow curvature leads to two large circulation cells towards the side walls then blockage and stagnation effects due to the pedestals. Away from the feed-hole the flow is more settled and evenly distributed, it shows a repeating pattern downwards. Results are generally in close agreement with the measured data. It was shown that the RANS models, in particular the SST model, perform better with high grid resolution in the impingement region. The velocity results showed that the $k-\varepsilon$ model failed to capture the impinging flow correctly due to the use of basic wall function and coarse mesh resolution while the rest of the models (realizable, SST) are closer to the LES and the experiment due to the use of more enhanced wall treatment and better grid resolution. The pedestal SGS approach was used to model the pedestal cooling tile, it showed close agreement with the CFD and the experiment especially in the developed area where the flow is independent of the feed-hole. The SGS model has much potential for further development to overcome the flow features in the impingement zone. In order to capture the correct flow pattern in the impingement region the first two rows were resolved with the mesh and combined with the SGS modelling of the rest of the tile, this gave optimum results of pressure, velocity and turbulence kinetic energy distribution. This study showed that the SGS pedestal model reduces the computation time by almost half the time needed to fully resolve the combustor pedestal tile.

\section{Acknowledgements}

This work was carried out within DYNAMO Design methods for durability and operability of low emission combustors, funded by the EU Clean Sky JTI-CS-2013-1-SAGE06-005. The authors would like to acknowledge the support of Rolls-Royce PLC. and the UTC group at Loughborough University which contributed to the accomplishment of this work. The authors would also like to acknowledge the support from Marco Zedda and Max Stauffer.

\section{References}

[1] M. S. Anand, R. Eggels, M. Staufer, M. Zedda, and J. Zhu. An Advanced Unstructured-Grid Finite-Volume
Design System for Gas Turbine Combustion Analysis. In ASME, Karnataka, India, dec 2013.

[2] P. A. Durbin. On the k-3 Stagnation Point Anomaly. International Journal of Heat and Fluid Flow, 17(1):8990, feb 1996.

[3] J. H. Ferziger and M. Peric. Computational Methods for Fluid Dynamics. Springer, 3rd editio edition, 2002.

[4] F. Haselbach and R. Parker. Hot End Technology for Advanced, Low Emission Large Civil Aircraft Engines. In 28th International Congress of the Aeronautical Sciences, Brisbane, Australia, 2012.

[5] B. E. Launder and D. B. Spalding. The Numerical Computation of Turbulent Flows. Computer Methods in Applied Mechanics and Engineering, 3(2):269-289, mar 1974.

[6] A .H. Lefebvre and M. V. Herbert. Heattransfer processes in gasturbine combustion chambers. Proceedings of the Institution of Mechanical Engineers, 174(1960):463-478, jun 1960.

[7] J. K. Luff. Numerical prediction of flow, thermal and stress fields in gas turbine combustor components. $\mathrm{PhD}$ thesis, Loughborough University, 2003.

[8] J. K. Luff and J. J. McGuirk. Numerical Prediction of Combustor Heatshield Flow and Heat Transfer With Sub-Grid-Scale Modelling of Pedestals. In ASME, New Orleans, Louisiana, USA, jun 2001.

[9] FR Menter. Two-equation eddy-viscosity turbulence models for engineering applications. AIAA journal, 32(8):1598-1605, 1994.

[10] D. E. Metzger, R. A. Berry, and J. P. Bronson. Developing Heat Transfer in Rectangular Ducts With Staggered Arrays of Short Pin Fins. Journal of Heat Transfer, 104(4):700, nov 1982.

[11] D. A. Paul, A. G. Barker, J. W. Charith, and J. J. Mcguirk. Modelling and Measurements of Combustor Cooling Tile Flows. In ASME, Atlanta, Georgia, USA, 2003.

[12] M. Prithiviraj and M. J. Andrews. Three Dimensional Numerical Simulation of Shell-and-tube Heat Exchangers. Part I: Foundaton and Fluid Mechanics. Numerical Heat Transfer, Part A: Applications, 33(8):799-816, jun 1998.

[13] T. H. Shih, W. W. Liou, A. Shabbir, Z. Yang, and J. Zhu. A New K-epsilon Eddy Viscosity Model for High Reynolds Number Turbulent Flows: Model Development and Validation. Technical report, NASA, aug 1994.

[14] J. Smagorinsky. General circulation experiments with the primitive equations. Monthly weather review, 91:99-164, 1963.

[15] S. J. Thorpe. Project 6 : Enhanced Cooling Systems for Low Emissions Combustors Dense Pedestal Cooling Tiles. Technical Report June, Loughborough University, 2008.

[16] A. A. Zukauskas. Heat Transfer from Tubes in Cross Flow. Advances in heat transfer, Academic Press, 8:93106, 1972. 